\title{
LEAST SQUARES SUPPORT VECTOR MACHINE MODEL FOR COORDINATE TRANSFORMATION
}

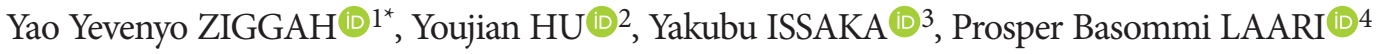 \\ ${ }^{1}$ Department of Geomatic Engineering, Faculty of Mineral Resources Technology, \\ University of Mines and Technology, Tarkwa, Ghana \\ ${ }^{2}$ Department of Surveying and Mapping, School of Information Engineering, \\ China University of Geosciences, Wuhan, P. R. China \\ ${ }^{3}$ Department of Geomatic Engineering, Faculty of Mineral Resources Technology, \\ University of Mines and Technology, Tarkwa, Ghana \\ ${ }^{4}$ Department of Environment and Resource Studies, University for Development Studies, Wa, Ghana
}

Received 10 October 2018; accepted 07 March 2019

\begin{abstract}
In coordinate transformation, the main purpose is to provide a mathematical relationship between coordinates related to different geodetic reference frames. This gives the geospatial professionals the opportunity to link different datums together. Review of previous studies indicates that empirical and soft computing models have been proposed in recent times for coordinate transformation. The main aim of this study is to present the applicability and performance of Least Squares Support Vector Machine (LS-SVM) which is an extension of the Support Vector Machine (SVM) for coordinate transformation. For comparison purpose, the SVM and the widely used Backpropagation Neural Network (BPNN), Radial Basis Function Neural Network (RBFNN), 2D conformal and affine methods were also employed. To assess how well the transformation results fit the observed data, the root mean square of the residual horizontal distances and standard deviation were used. From the results obtained, the LS-SVM and RBFNN had comparable results and were better than the other methods. The overall statistical findings produced by LS-SVM met the accuracy requirement for cadastral surveying applications in Ghana. To this end, the proposed LS-SVM is known to possess promising predictive capabilities and could efficiently be used as a supplementary technique for coordinate transformation.
\end{abstract}

Keywords: coordinate transformation, Support vector machine, Least squares support vector machine, 2D conformal model, 2D affine model.

\section{Introduction}

Over the years, the means to facilitate the usage of Global Navigation Satellite System (GNSS) acquired data in countries utilising astro-geodetic datums has been a major research focus in the geodetic community. Reliable estimation of transformed coordinates between global and local datums is one of the fundamental problems in geodetic sciences. Several authors have attributed such problems to the (i) data collection procedures employed when establishing the local geodetic datum; (ii) computational adjustment techniques applied to adjust and unify the local geodetic reference networks; (iii) the quality of the data set collected in the local geodetic reference network; and (iv) lack of ellipsoidal height for the local geodetic datum
(Tierra, Dalazoana, \& De Freitas, 2008; Yang, 2009; Varga, Grgić, \& Bašić, 2015). These challenges have therefore contributed to the heterogeneity in data relating to the local geodetic networks. Countries that are yet to establish and migrate onto the geocentric datum are confronted with the above-mentioned concerns. To circumvent the situation, conventional transformation techniques which are parametric and thus require a fixed functional form to the co-located point coordinates have been extensively used for two and three-dimensional coordinate transformation in different geodetic reference networks across the globe (see e.g. Varga et al., 2015 and references therein). The conventional transformation methods could be categorised into conformal (e.g. Bursa-Wolf, Molodensky-Badekas, Abridged Molodensky, Veis, 2D conformal), affine

${ }^{*}$ Corresponding author. E-mail: zhuzhenjun@seu.edu.cn 
(e.g. 2D affine, 8-parameter, 9-parameter, 12-parameter) and projective (e.g. 2D and 3D projective).

In the past few years, several computational intelligent techniques have been developed and applied to solve different problems in geodesy and geosciences. Artificial Neural Network (ANN) is one of the most attractive methods of computational intelligence to cope with non-linearity and time varying geodetic data due to its ability to learn and adapt to new dynamic environments. Numerous studies have shown successful implementation of ANN in the geodetic disciplines including but are not limited to deformation studies (Li \& Kong, 2014; Huang, Wu, \& Ziggah, 2016), meteorological studies (Mohammadi et al., 2015; Durmaz \& Karslioglu, 2011), hydrological studies (Tiwari, J. Adamowski, \& K. Adamowski, 2016; Deo \& Şahin, 2016; Deo, Tiwari, Adamowski, \& Quilty, 2017), tidal estimation (Okwuashi \& Ndehedehe, 2017), change detection (Pal, 2009; Chang, Han, Yao, Chen, \& $\mathrm{Xu}, 2010$ ), geoid determination (Kavzoglu \& Saka, 2005; Sorkhabi, 2015), and gravity field modelling (Turgut, 2016). Additionally, extensive studies on the suitability of ANN for coordinate transformation in both $2 \mathrm{D}$ and $3 \mathrm{D}$ have also been duly investigated by several authors (Tierra et al., 2008; Zaletnyik, 2004; Lin \& Wang, 2006; Tierra, De Freitas, \& Guevara, 2009; Tierra \& Romero, 2014; Gullu, 2010; Gullu et al., 2011; Turgut, 2010; Mihalache, 2012; Yilmaz \& Gullu, 2012; Konakoğlu, Cakir, \& Gökalp, 2016; Konakoğlu \& Gökalp, 2016; Ziggah, Youjian, Tierra, Konate, \& Hui, 2016; Kumi-Boateng \& Ziggah, 2017). A thorough review of these coordinate transformation studies indicates that the ANN of radial basis function and backpropagation have been the most commonly used techniques. The main objective noted from the review was about testing the applicability of ANN in coordinate transformation. It was noticed that, the ANN can produce improved transformation results when compared with the conventional transformation methods. The main advantage of the ANN has been attributed to its non-parametric characteristics. That is, the ANN is capable of approximating dataset without prior knowledge of the functional relationship and data distribution between the co-located points. This has further confirmed the proofs made by Hornik, Stinchcombe, and White (1989) and Park and Sandberg (1991) that, ANN could be regarded as a universal function approximator. Another significance of the ANN lies in its ability to not rely on transformation parameters due to its adaptive computational scheme. That is, once the optimum trained model has been developed, prediction can be made when unseen dataset is introduced into the network.

In addition to the proficiencies of ANN, recent research works have led to finding and testing new alternative computational intelligent methods. In recent years, Least Squares Support Vector Machine (LS-SVM) which is an extension of Support Vector Machine (SVM) has been one of the most significant computational intelligent theories introduced for classification and function estimation tasks. LS-SVM has been shown in various studies to exhibit the major advantages of global optimum realisation, higher generalisation performance and good computational efficiency. In addition, the LS-SVM technique only learns on fewer training data to produce the optimum trained model. Hence, it can be used to fit a model in scarcity of data situation. These derived computational merits from LS-SVM can be confirmed in a number of studies found in the geodetic sciences domain (see e.g. Li \& Kong, 2014; Mohammadi et al., 2015; Durmaz \& Karslioglu, 2011; Okwuashi \& Ndehedehe, 2015, 2017 and references therein).

Despite the fact that LS-SVM has been applied in several disciplines and to solve some geodetic problems, its application in coordinate transformation is still very rare in literature. In view of that, the present study seeks to ascertain the extent of applicability and performance of LS-SVM in coordinate transformation. A comparison with SVM and the widely used Backpropagation Neural Network (BPNN), Radial Basis Function Neural Network (RBFNN) and two conventional transformation techniques namely, $2 \mathrm{D}$ conformal and $2 \mathrm{D}$ affine model was carried out accordingly. The numerical case study application is Ghana located in the Western part of Africa. In Ghana two horizontal geodetic datums (Accra and Leigon) are being utilised for its mapping and surveying purposes. Due to data incompatibility between the two local geodetic datums, transformation of coordinates is necessary. The authors believe that it will be an excellent opportunity to gain insight into the viability of LS-SVM for coordinate transformation. The entire present study forms part of a larger effort by the Ghana Survey and Mapping Division of Lands Commission towards the adoption and implementation of computational intelligent procedures to transform coordinates in Ghana. The study will help Land surveyors and other built environment practitioners in Ghana to know the efficiency of using such intelligent tools and applying them accordingly.

The paper is organized as follows. Sections 1 and 2 present the study area and the data set used. Section 3 briefly presents the theoretical concept of the vector machine methods introduced. Section 4 gives the model adequacy assessments for the various methods, while Section 5 discusses the results. The study ends with conclusions.

\section{Study area}

Ghana geodetic reference network (Figure 1) is the focus of this study. Ghana is a country located in the Western part of Africa that shares border with Ivory Coast in the West, Togo to the East, Burkina Faso to the North and Gulf of Guinea to the South. Ghana has a land area of 238, $540 \mathrm{~km}^{2}$ (Fosu, Poku-Gyamf, \& Hein, 2006) and lies between latitudes $4^{0} 30^{\prime} \mathrm{N}$ and $11^{0} \mathrm{~N}$, and between longitudes $3^{0} \mathrm{~W}$ and $1^{0} \mathrm{E}$ (Mugnier, 2000). In Ghana, two local geodetic datums are defined for surveying and mapping purposes: the Accra 1929 and Leigon 1977 (Ayer \& Fosu, 2008; Poku-Gyamfi, 2009; Ayer, 2008; Kotzev, 2013). The Accra 1929 datum is the local realisation of the War 
Office 1926 ellipsoid, while the Leigon datum is the realisation of the Clark 1880 (modified) ellipsoid (Ayer \& Fosu, 2008; Ayer, 2008). The War Office 1926 ellipsoid has a semi-major axis (a) = $6378299.99899832 \mathrm{~m}$, semi-minor axis (b) = $6356751.68824042 \mathrm{~m}$ and flattening (f) $0=$ $1 / 296$. The Clark 1880 (modified) has the following ellipsoid properties: semi-major axis (a) =6 $378249.145 \mathrm{~m}$, semi-minor axis (b) = $6356514.870 \mathrm{~m}$ and flattening $\mathrm{f}=$ $1 / 293.465006079115$.

The coordinate system approved for geospatial works in Ghana is the projected grid coordinates based on the Transverse Mercator $1^{\circ} \mathrm{NW}$. The origin of the Transverse Mercator is longitude $1^{\circ} 00^{\prime} \mathrm{W}$ (central meridian) and latitude $04^{\circ} 40^{\prime} \mathrm{N}$ with $274319.736 \mathrm{~m}$ as the false Easting added to all Y coordinates to avoid negative coordinates and the false Northing set to zero. A scale factor of 0.99975 is used at the central meridian so that the scale distortion exceeds the projection values only at the extreme ends of the country (Mugnier, 2000).

\section{Data source}

In this study, 2D coordinate transformation was conducted using planimetric data of 46 co-located reference stations (Figure 1). These stations are in projected grid coordinate system (Eastings and Northings) based on the
Transverse Mercator for the Accra 1929 and Leigon 1977 obtained from the Ghana Survey and Mapping Division of Lands Commission. These datasets belong to the Ghana National Triangulation Network of points (Figure 1).

\section{Methods}

The main focus here is to present a brief account on the SVM and LS-SVM introduced in this study. The BPNN, RBFNN, 2D conformal and 2D affine mathematical backgrounds and theories will not be repeated here. These have been successfully and frequently applied in coordinate transformation. Therefore, a more comprehensive detail on them can be found in (Bishop, 1995; Haykin, 1990; Ghilani, 2010).

\subsection{Support Vector Machine}

SVM can be applied to solve pattern recognition (Vapnik, 1998) and function estimation (Drucker, Burges, Kaufman, Smola, \& Vapnik, 1997) problems with the latter being the main focus of this study. In the SVM function estimation, given a training data set of input vectors $X=\left\{x_{n}\right\}$ with related target values $Y=\left\{y_{n}\right\}, n=1 \ldots N$, the objective is to define a functional model $y(x)$ that can predicts satisfactory $y$ values given $x$. Here, the $N$-dimensional input vector

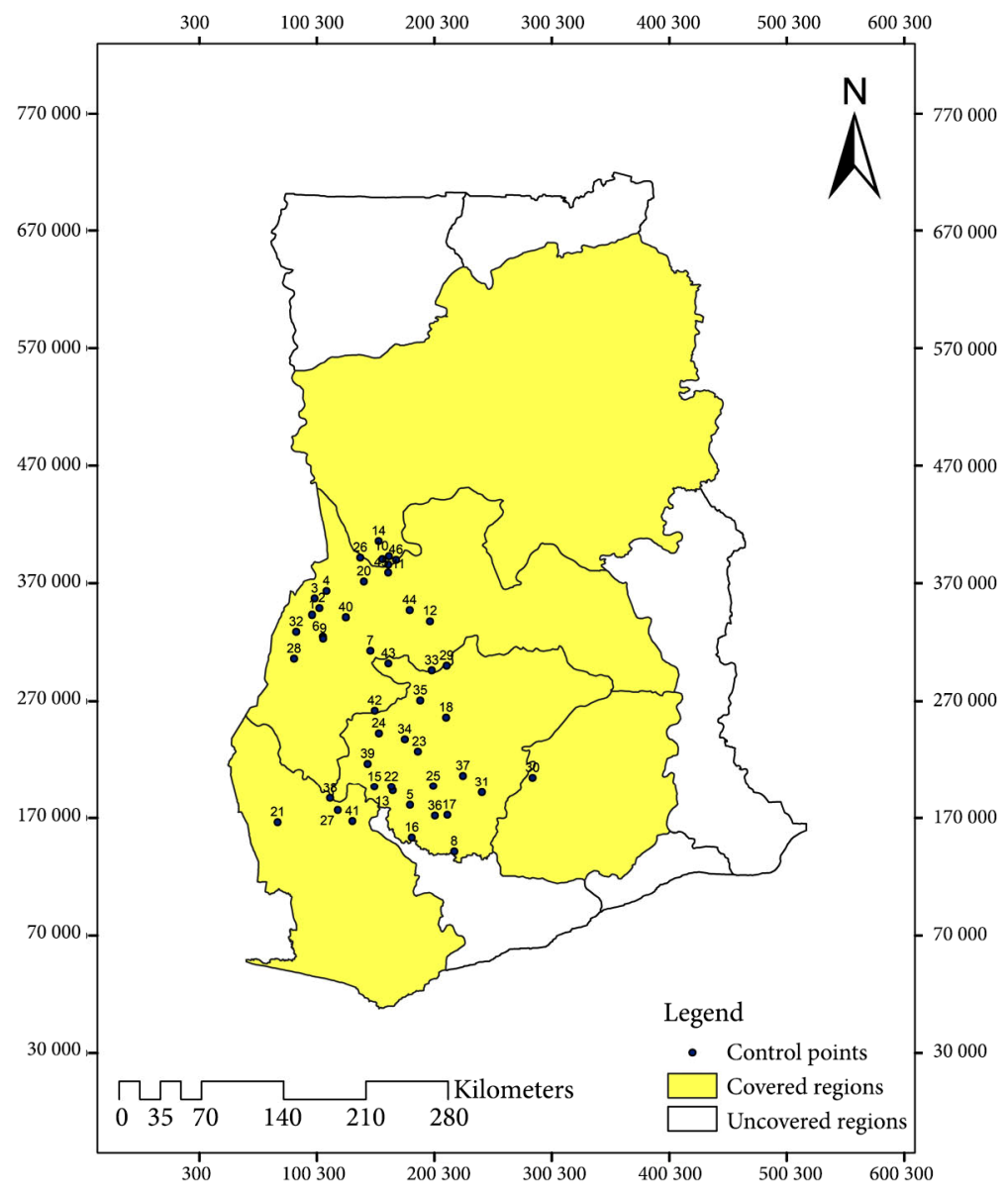

Figure 1. Study area: distribution of data points 
$X$ is mapped from a low-dimensional space into a higher dimensional feature space using the nonlinear function $\varphi(x)$ (Equation (1)). A linear model (Ao \& Palade, 2011; Smola \& Schölkopf, 2004) expressed in Equation (1) is then built in this high-dimensional feature space.

$$
y(x)=w^{T} \varphi\left(x_{i}\right)+b,
$$

where $w=\left(w_{1}, w_{2}, \ldots, w_{M}\right)$ is the weight vector, $\phi(x)=\left(\phi\left(x_{1}\right), \ldots, \varphi\left(x_{i}\right)\right)$ is the basis function vector denoting a set of nonlinear transformations, and $b$ the bias term. It is accepted that the objective of SVM is to determine the optimal separating hyperplane that maximizes the margin of the training data. In order to find the optimal hyperplane, a quadratic programming optimization problem will have to be solved. In lieu of that, the SVM fit a function to the training data by solving the optimization problem with constraints. The SVM constrained quadratic programming (Xiang-Yang, Jing-Wei, \& Hong-Ying, 2011) can be represented by Equation (2) subject to Equation (3) as

$$
\begin{gathered}
\min _{w, b, \xi, \xi^{*}} \frac{1}{2} w^{T} w+C \sum_{i=1}^{n}\left(\xi+\xi_{i}^{*}\right) ; \\
w^{T} \phi\left(x_{i}\right)+b-y_{i} \leq \varepsilon+\xi_{i} ; \\
y_{i}-w^{T} \phi\left(x_{i}\right)-b \leq \varepsilon+\xi_{i}^{*} ; \\
\xi, \xi_{i}^{*} \geq 0, i=1, \ldots, n,
\end{gathered}
$$

where the constant $C$ which varies in the interval $0<C<\infty$ is a pre-defined penalty value that regulates the error, while $\xi, \xi_{i}{ }^{*}$ are the non-negative slack variables that give the degree of variation of training datasets outside $\varepsilon$-insensitive zone. $\varepsilon$ (Equation (3)) is the error parameter which limits the deviation between the fitted function model from the desired (target) values. Equation (2) subject to the constraints in Equation (3) could be solved using Lagrangian function and Lagrange multipliers (Xiang-Yang et al., 2011) as defined by Equation (4).

$$
\begin{aligned}
& L=\frac{1}{2}\left(w^{T} w\right)+C\left(\sum_{i=1}^{n} \xi_{i}^{*}+\sum_{i=1}^{n} \xi\right)- \\
& \sum_{i=1}^{n} \alpha_{i}^{*}\left(y_{i}-w^{T} \phi\left(x_{i}\right)-b+\varepsilon+\xi_{i}^{*}\right)- \\
& \sum_{i=1}^{n} \alpha_{i}\left(w^{T} \phi\left(x_{i}\right)+b-y_{i}+\varepsilon+\xi_{i}\right)-\sum_{i=1}^{n}\left(\gamma_{i}^{*} \xi_{i}^{*}+\gamma_{i} \xi_{i}\right),
\end{aligned}
$$

where $L$ is the Lagrangian and $\gamma_{i}, \gamma^{*}, \alpha_{i}, \alpha^{*}$ are the Lagrange multipliers. The solution of Equation (4) must satisfy the Karush-Kuhn-Tucker (KKT) conditions (Farag \& Mohamed, 2004). Hence, Equation (2) is reduced into a dual optimization problem expressed in Equation (5) as maximize

$$
\begin{array}{r}
-\frac{1}{2} \sum_{i, j}^{n}\left(\alpha_{i}-\alpha_{i}^{*}\right)\left(\alpha_{j}-\alpha_{j}^{*}\right) M_{i j}- \\
\quad \varepsilon \sum_{i=1}^{n}\left(\alpha_{i}+\alpha_{i}^{*}\right)+\sum_{i=1}^{n} y_{i}\left(\alpha_{i}-\alpha_{i}^{*}\right)
\end{array}
$$

with the constraints (Equation (6))

$$
\sum_{i}^{n}\left(\alpha_{i}-\alpha_{i}^{*}\right)=0 \text { and } \alpha_{i}, \alpha_{i}^{*} \in[0, C],
$$

where $M_{i j}=K\left(x_{i}, x_{j}\right)=\phi\left(x_{i}\right)^{T} \phi\left(x_{j}\right)$ is the kernel trick (Mercer theorem). It is important to note that the dual variables in Equation (4) satisfy the positivity constraints that is $\gamma_{i}, \gamma^{*}, \alpha_{i}, \alpha^{*} \geq 0$. The $\alpha_{i}$ and $\alpha_{i}^{*}$ are then computed and the optimum weight vector $(w)$ of the function estimation model hyperplane is given by Equation (7) as

$$
w=\sum_{i=1}^{n}\left(\alpha_{i}-\alpha_{i}^{*}\right) \phi\left(x_{i}\right) .
$$

Therefore, the SVM function estimation model applied in this study for the coordinate transformation can be expressed in the form of Equation (8).

$$
y(x)=\sum_{i=1}^{n}\left(\alpha_{i}-\alpha_{i}^{*}\right) K\left(x, x_{i}\right)+b .
$$

Here, $K\left(x, x_{i}\right)$ is the kernel function. In this study, the Gaussian kernel, polynomial kernel, sigmoid kernel and linear kernel were applied on the dataset with the objective of selecting the kernel function that maps the training data patterns more effectively. After several trials, the polynomial kernel function fitted the pattern of the data accurately than the other kernel methods. Therefore, the polynomial kernel function (Güraksin, Hakli, \& Harun, 2014) adopted in this study is given by Equation (9).

$$
K\left(x_{i}, x_{j}\right)=\left(1+x_{i}^{T} x_{j}\right)^{q} .
$$

\subsection{Least Squares Support Vector Machine}

LS-SVM proposed by Suykens, Van Gestel, De Brabanter, De Moor, and Vandewalle (2002) is the least squares formulation of SVM and is applied to solve classification and function estimation related problems. In this study, the LS-SVM was formulated as a function estimation technique to carry out the coordinate transformation. Given a set of training data $D=\left\{\left(x_{m}, y_{m}\right) \mid m=1,2, \ldots, n\right\}$ with input data $x_{m} \in R^{n}$ and corresponding target $y_{m} \in r$, where $R^{n}$ is the $n$-dimensional vector space and $r$ the one-dimensional vector space. The objective is to fit a functional model $y(x)$ on the training data sets such that this function could be used later on to infer the target $y$ for a new input data point $x$. The LS-SVM model can be expressed in the form of Equation (10) as

$$
y(x)=w^{T} \phi(x)+b,
$$

where $w$ is the adjustable weight vector, $T$ is the transpose, $\phi(x)$ is the nonlinear transformation that maps the input data into a higher dimensional space and $b$ is the scalar threshold.

In line with the structural risk minimization rule (Kecman, 2001), the risk bound is minimized by devising the following optimization problem (Suykens \& Vandewalle, 1999) expressed in Equation (11) with the equality constraints given in Equation (12). 


$$
\begin{aligned}
& \operatorname{minimize} \frac{1}{2} w^{T} w+\gamma \frac{1}{2} \sum_{m=1}^{n} e_{m}^{2} ; \\
& y(x)=w^{T} \phi\left(x_{m}\right)+b+e_{m} .
\end{aligned}
$$

Here, $\gamma$ is the regularization parameter that determines the tradeoff between the fitting function error minimization and flatness, $e_{m}$ is the error variable and $m=$ $1, \ldots, n$.

In order to solve Equation (11) subject to Equation (12), Lagrange function $(L)$ defined in Equation (13) is used.

$$
\begin{aligned}
& L(w, b, e, \alpha)=\frac{1}{2} w^{T} w+ \\
& \gamma \frac{1}{2} \sum_{m=1}^{n} e_{m}^{2}-\sum_{m=1}^{n} \alpha_{m}\left\{y_{m}\left(w^{T} \phi\left(x_{m}\right)+b\right)-1+e_{m}\right\} .
\end{aligned}
$$

Here, $\alpha_{m}$ values are the Lagrange multipliers which could be positive or negative due to the equality constraints. The optimality condition of Equation (13) was met by finding the partial derivative of $L$ with respect to each variable as provided in Equation (14).

$$
\begin{aligned}
& \frac{\partial L}{\partial w}=0 \Rightarrow w=\sum_{m=1}^{n} \alpha_{m} \phi\left(x_{m}\right) \\
& \frac{\partial L}{\partial b}=0 \Rightarrow \sum_{m=1}^{n} \alpha_{m}=0 ; \\
& \frac{\partial L}{\partial e_{m}}=0 \Rightarrow \alpha_{m}=\gamma e_{m}, \forall m=1, \ldots, n ; \\
& \frac{\partial L}{\partial \alpha_{m}}=0 \Rightarrow w^{T} \phi\left(x_{m}\right)+b+e_{m}-y_{m}=0, \forall m=1, \ldots, n .
\end{aligned}
$$

Let $D^{T}=\left[\phi\left(x_{1}\right)^{T} y_{1}, \ldots, \phi\left(x_{n}\right)^{T} y_{n}\right], \quad y=\left[y_{1}, \ldots, y_{n}\right]$, $1_{v}=[1, \ldots, 1], e=\left[e_{1}, \ldots, e_{n}\right], \alpha=\left[\alpha_{1}, \ldots, \alpha_{n}\right]$. Eliminating $w$ and $e$ parameters, one obtains the following linear Karush-Kuhn-Tucker (KKT) system given by Equation (15) (Suykens et al., 2002).

$$
\left[\frac{0}{y} \mid \frac{y^{T}}{\Omega+I / \gamma}\right]\left[\frac{b}{\alpha}\right]=\left[\frac{0}{1_{v}}\right] \text {, solving in } \alpha \text { and } b,
$$

where $D^{T} D$ and the kernel trick (Mercer's theorem) (Vapnik, 1998) was applied within the $\Omega$ matrix represented in Equation (16) as

$$
\Omega_{m l}=y_{m} y_{l} \phi\left(x_{m}\right)^{T} \phi\left(x_{l}\right)=y_{m} y_{l} K\left(x_{m}, x_{l}\right), \forall m, l=1, \ldots, n \text {. }
$$

Here, $K\left(x_{m}, x_{l}\right)$ is the kernel function. Among the kernel functions available, the radial basis function produced the best results. Therefore, the present study applied the radial basis function (Gencoglu \& Uyar, 2009) expressed in Equation (17) as

$$
k\left(x_{m}, x_{l}\right)=\exp \left\{-\frac{\left(x_{m}-x_{l}\right)\left(x_{m}-x_{l}\right)^{T}}{2 \sigma^{2}}\right\},
$$

where $\sigma$ is the width of the radial basis function. The resulting LS-SVM function estimation model for conducting the coordinate transformation is defined in Equation (18).

$$
y(x)=\operatorname{sign}\left[\sum_{m=1}^{n} \alpha_{m} y_{m} K\left(x, x_{m}\right)+b\right] .
$$

\section{Model quality evaluation}

The differences between the observed and transformed coordinates were used to evaluate the methods applied performance. To do that, horizontal error (HE), minimum horizontal error, maximum horizontal error, average horizontal error (AHE), standard deviation (SD) and root mean square of the horizontal residual distances $\left(\mathrm{RMS}_{\mathrm{HE}}\right)$ were utilised. Equations (19) to (23) present their mathematical notations.

$$
\begin{aligned}
& H E=\sqrt{\left(E_{O_{i}}-E_{P_{i}}\right)^{2}+\left(N_{O_{i}}-N_{P_{i}}\right)^{2}}=\sqrt{\Delta E^{2}+\Delta N^{2}} \\
& \text { Max Error }=\max (H E)_{i=1}^{n} ; \\
& A H E=\frac{1}{n} \sum_{i=1}^{n} H E_{i} ; \\
& S D=\sqrt{\frac{1}{n-1} \sum_{i=1}^{n}(H E-\bar{H} \bar{E})^{2}} \\
& R M S_{H E}=\sqrt{\frac{\sum\left(H E_{i}\right)^{2}}{N}}
\end{aligned}
$$

Here, $n$ is the total number of observations and $\bar{H} \bar{E}$ is the mean of the horizontal error. $\left(E_{O}, N_{O}\right)$ are the observed coordinates and $\left(E_{P}, N_{P}\right)$ are the transformed coordinates from the various procedures.

\section{Application}

\subsection{Model formulation}

In order to implement the various methods (LS-SVM, SVM, BPNN, RBFNN, 2D affine and 2D conformal), the 46 co-located reference control points found in the two horizontal geodetic datums (Accra and Leigon) in Ghana were used. Here, the objective is to transform coordinates from the Leigon 1977 to the official Accra 1929 datum. Plane projected grid coordinates of Easting (E) and Northing (N) related to the Leigon 1977 datum were used as the input data. The Accra 1929 datum coordinates were used as the target data in the model formulation process. The input data in this study was denoted as $\left(E_{\text {clark }}, N_{\text {clark }}\right)$ while the target (output) was represented as $\left(E_{\text {war }}, N_{\text {war }}\right)$, respectively. The study data set was first divided into training and testing. The training data was used to build the coordinate transformation models derived from the various methods applied. The testing data was introduced into the selected optimum trained models for performance evaluation. In this study, $67 \%$ of the entire data representing 31 co-located points constituted the training set while the other 33\% making 15 co-located points formed the testing set. The 


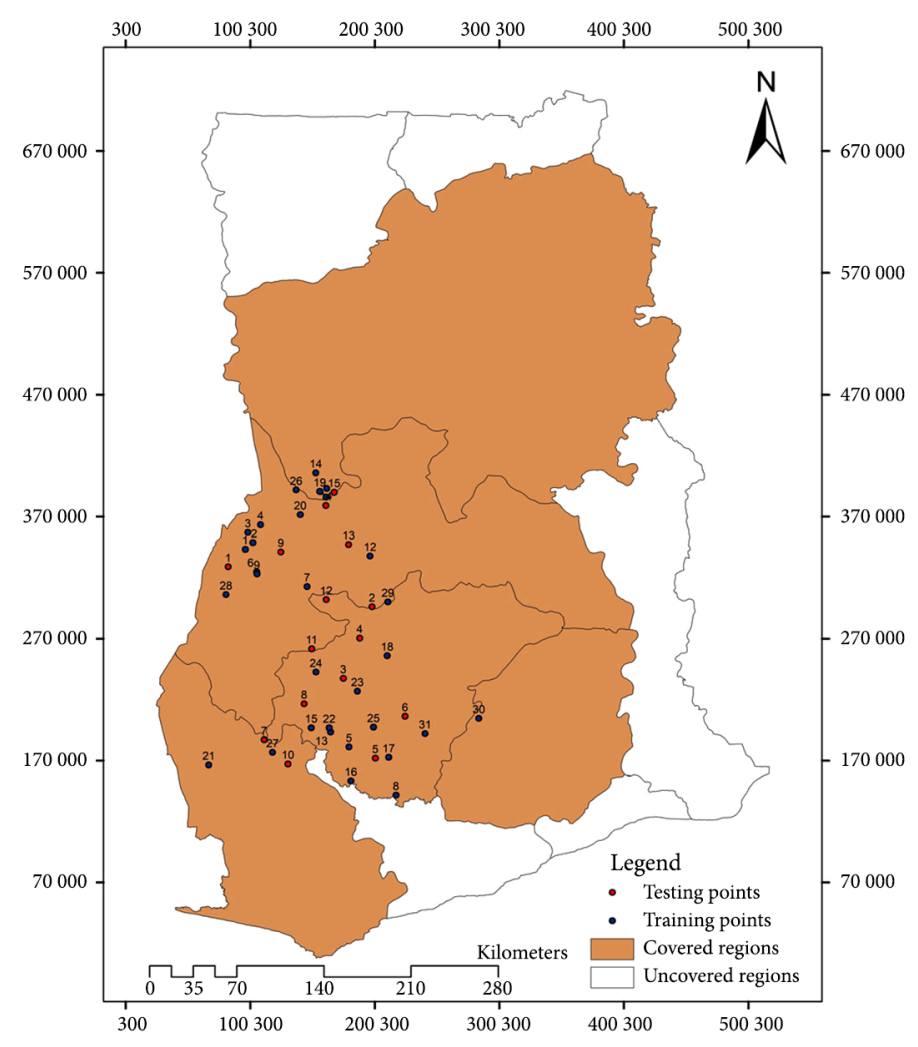

Figure 2. Training and testing data distribution

training data was carefully selected to capture the area of interest as shown in Figure 2.

The idea behind these training points selection was to develop a model that could be applied in any of the regions covered by the entire study data set. This will help provide a better idea on the data adaptation strength for each method when performing 2D coordinate transformation in the study area. Correspondingly, an evenly distributed points is also selected to constitute the test data. In doing so, the extent of application and accuracy level for each developed transformation model within the five regions of study (Figure 2) will be clearly known. Figure 2 shows a spatial map of the geographical distributions of the selected training and testing points.

Due to different physical realisations among the input data sets, data normalisation was carried out. The normalisation process create uniformity among the input data sets thereby contributing to the successful implementation of the computational intelligent algorithms. In effect, large values will not have much influence on the smaller values. Therefore, for the model training, the study data set was normalised between -1 and 1 using Equation (24).

$$
t_{i}=t_{\min }+\frac{\left(t_{\max }-t_{\min }\right) \times\left(b_{i}-b_{\min }\right)}{\left(b_{\max }-b_{\min }\right)},
$$

where $t_{i}$ represents the normalised data, $b_{i}$ is the observed coordinates, while $b_{\min }$ and $b_{\max }$ represents the minimum
Table 1. 2D affine model transformation parameters from Leigon 1977 to Accra 1929 datum

\begin{tabular}{|c|c|c|}
\hline Parameters & Values $(\mathrm{m})$ & SD $(\mathrm{m})$ \\
\hline A & 1.00001260 & $2.38 \mathrm{E}-06$ \\
\hline B & $1.36 \mathrm{E}-05$ & $1.36 \mathrm{E}-06$ \\
\hline C & 0.5414654 & 0.6367 \\
\hline D & $-5.39 \mathrm{E}-06$ & $2.38 \mathrm{E}-06$ \\
\hline E & 1.00001 & $1.36 \mathrm{E}-06$ \\
\hline F & -2.3336951 & 0.6367 \\
\hline
\end{tabular}

Table 2. 2D conformal model transformation parameters from Leigon 1977 to Accra 1929 datum

\begin{tabular}{|c|c|c|}
\hline Parameters & Values $(\mathrm{m})$ & SD $(\mathrm{m})$ \\
\hline $\mathrm{a}$ & 1.00001 & $1.13 \mathrm{E}-06$ \\
\hline $\mathrm{b}$ & -0.00001 & $1.13 \mathrm{E}-06$ \\
\hline $\mathrm{c}$ & 1.56449 & 0.37599 \\
\hline $\mathrm{d}$ & -1.10457 & 0.37599 \\
\hline
\end{tabular}

and maximum value of the observed coordinates with $t_{\max }$ and $t_{\min }$ values set at 1 and -1 .

The performance of the optimum LS-SVM, SVM, BPNN and RBFNN models was ascertained using the root mean square error (RMSE) criterion. The RMSE value was estimated using Equation (25). 


$$
R M S E=\sqrt{\frac{1}{n} \sum_{i=1}^{n}\left(O_{i}-P_{i}\right)^{2}}
$$

where $O$ and $P$ are the observed and transformed coordinates. Here, the RMSE values for each training and testing phase were monitored for the LS-SVM, SVM, BPNN and RBFNN. Here, the trained model that furnished the lowest RMSE value closer to zero in the testing stage was selected as the optimum. These obtained RMSE results describe the ability of the applied methods to appropriately capture the input-output mapping relationship between the training inputs and target data sets.

In the course of the SVM training, different combinations of $\varepsilon$ (Equation (3)), $C$ (Equation (2)) and kernel functions were tried to get the best coordinate transformation results. For the target $\left(E_{\text {war }}, N_{\text {war }}\right)$ prediction, the optimum design values for $\varepsilon$ and $C$ was 0.00000001 and 50 . The polynomial kernel (Equation (9)) of order one was the best kernel in this regard. For the LS-SVM trained model, the optimum design values of $\gamma$ (Equation (11)) and $\sigma^{2}$
(Equation (17)) that produced the best transformed $E_{\text {war }}$ values are 6761965743036.15 and 1092.552454. In the case of $N_{\text {war }}$, the optimum design values for $\gamma$ and $\sigma^{2}$ was 1625293587807.38 and 1514173.748 , respectively. In the ANNs (BPNN and RBFNN) model building, the same data set used in the SVM and LS-SVM was applied. The optimum RBFNN model consisted of two inputs ( $E_{\text {clark }}$, $N_{\text {clark }}$ ), a single hidden layer with 18 neurons and two outputs $\left(E_{\text {war }}, N_{\text {war }}\right)$ that is, [2-18-2]. The BPNN had [2-8-1] for transforming $E_{w a r}$ and [2-11-1] for the $N_{w a r}$ output vector. This means that, the BPNN model for predicting the output $E_{\text {war }}$ comprises of two inputs with eight hidden neurons and the $N_{\text {war }}$ consisted of eleven hidden neurons with two inputs.

With regards to the $2 \mathrm{D}$ affine and conformal models, the training data set was used to derive the transformation parameters based on the least squares estimation principle. The obtained results are shown in Tables 1 and 2. The calculated SD values in Tables 1 and 2 offer indication on the precision extent of the derived transformation parameters.

Figures 3 and 4 show a diagrammatic representation of the RMSE training and testing results obtained for the best

a)

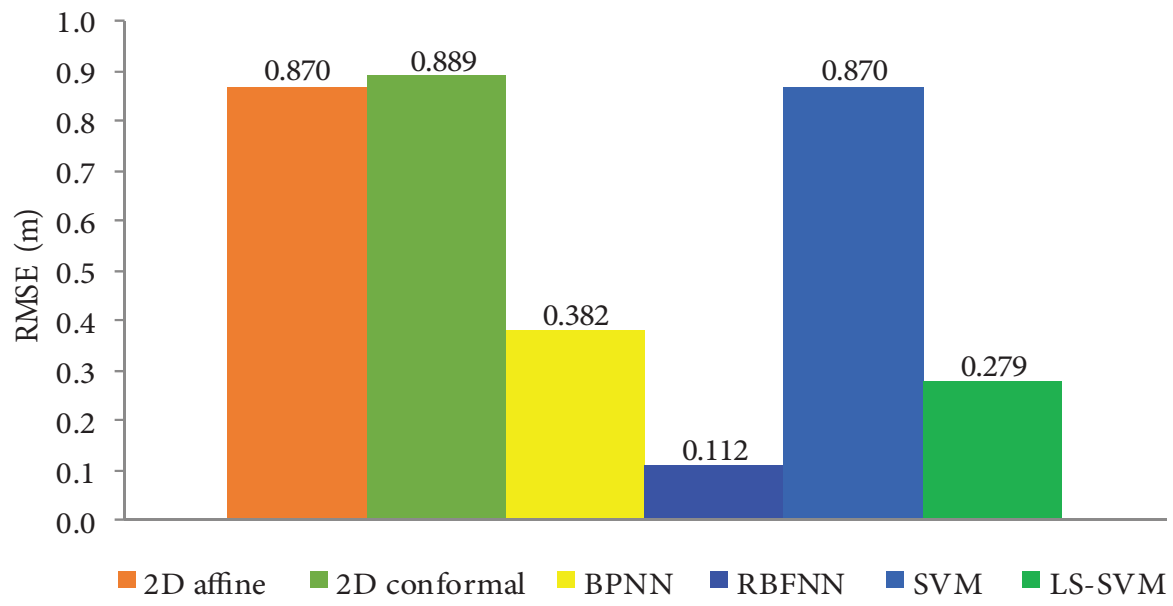

b)

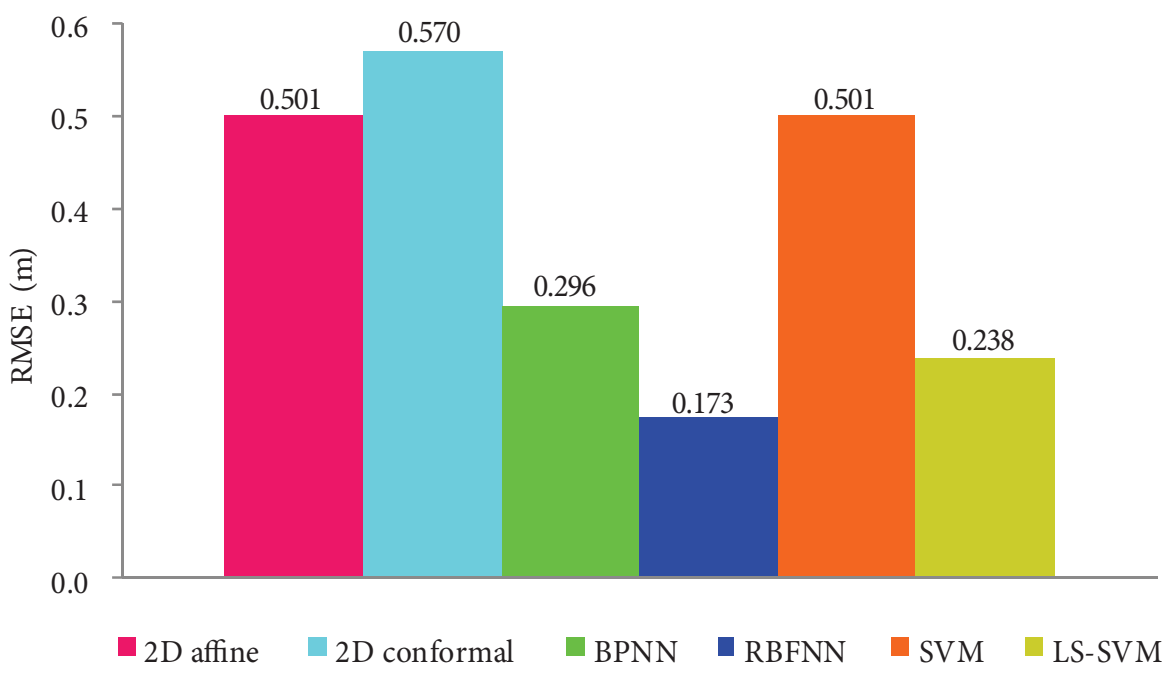

Figure 3. RMSE results of the methods based on the training data for (a) Easting and (b) Northing coordinates 
a)

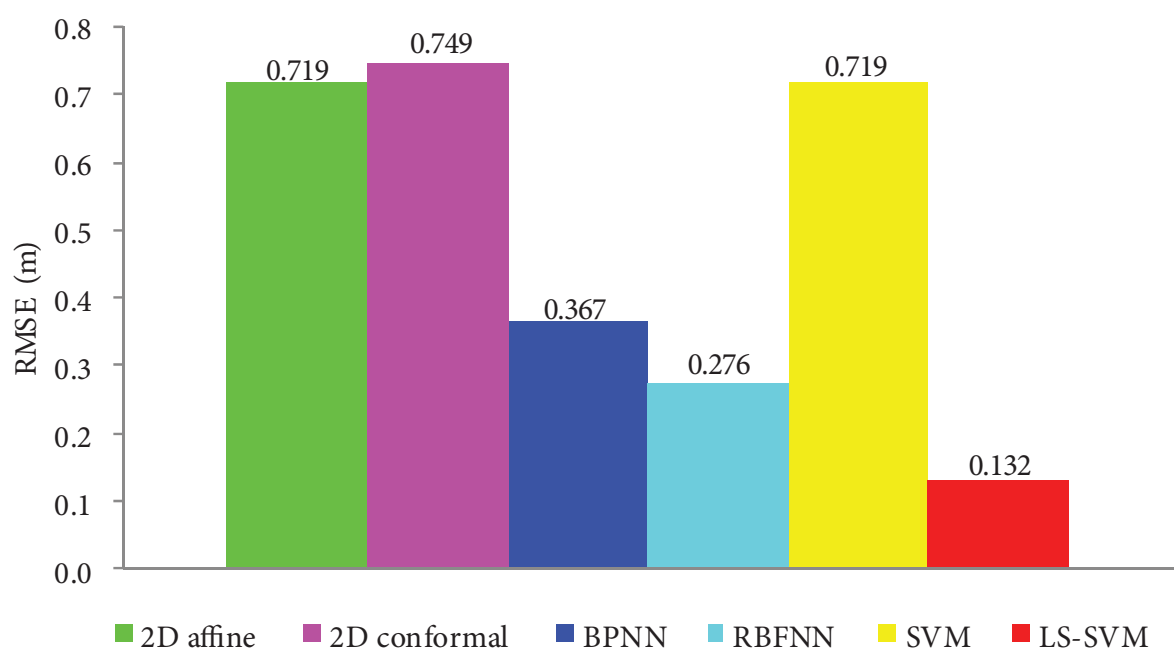

b)

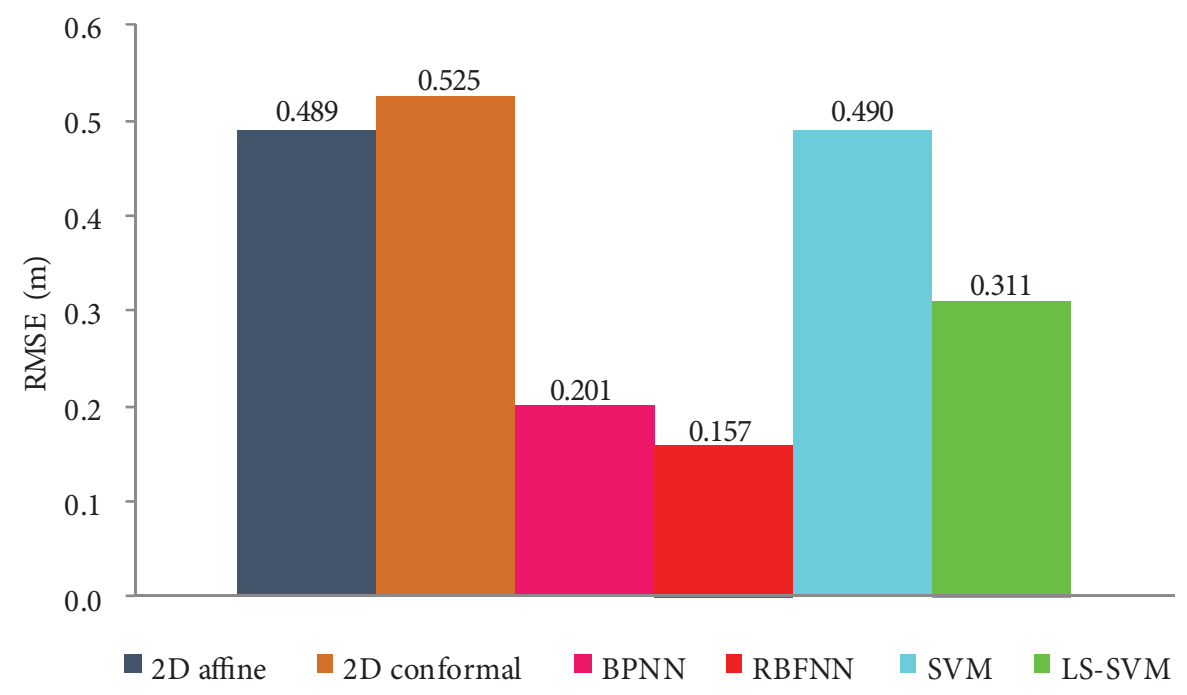

Figure 4. RMSE results of the methods based on the testing data for (a) Easting and (b) Northing coordinates

trained models. A visual observation of Figures 3 and 4 revealed marginal RMSE deviation when comparing each method training RMSE results against their corresponding testing results. This therefore suggests that none of the methods applied exhibited overfitting condition. Comparatively, the LS-SVM, RBFNN and, BPNN performed better than the SVM, 2D affine and conformal model (Figures 3 and 4). The interpretation that could be made here is that the LS-SVM, RBFNN and BPNN in training have demonstrated greater calibration power and in testing were able to give good generalisation performance. The assertion is in line with the rule of thumb that, the closer the RMSE is to zero the better the model predictions in consonance with the observed data.

\subsection{Test results}

Mathematically, the test results produced will provide the model developer and the user a better idea of all possible disparities in the transformed coordinates. Consequently, an indication on the generalisation strength and predictive potential of the techniques employed shall be known for the study area. Table 3 shows how much the predicted horizontal test coordinates produced by the various methods deviated from the observed coordinates. The obtained horizontal positional error (HE) which was estimated using Equation (19) shows the prediction limitations of the methods and thus signify the extent at which their results are in conformance with the observed coordinates.

When the test results presented in Table 3 are analysed, it can be noticed that the LS-SVM, BPNN and RBFNN were able to produce horizontal coordinate residuals below $1 \mathrm{~m}$ as compared with the SVM, 2D affine and conformal models having values above $1 \mathrm{~m}$. This can additionally be confirmed in Figure 5 .

Therefore, the obtained results suggest that the proposed LS-SVM as well as the RBFNN and BPNN transformed the test coordinates with a significantly better accuracy than the other models. The inability of the SVM to produce better generalisation performance could be 
Table 3. Horizontal coordinate residuals from the various methods (unit: meters)

\begin{tabular}{|c|c|c|c|c|c|c|}
\hline Test Point ID & 2D Affine & 2D Conformal & SVM & BPNN & RBFNN & LS-SVM \\
\hline $\mathrm{R} 1$ & 1.618 & 1.519 & 1.618 & 0.306 & 0.285 & 0.416 \\
\hline $\mathrm{R} 2$ & 1.040 & 1.319 & 1.040 & 0.522 & 0.163 & 0.352 \\
\hline R3 & 0.614 & 0.665 & 0.614 & 0.052 & 0.143 & 0.114 \\
\hline $\mathrm{R} 4$ & 0.535 & 0.698 & 0.535 & 0.303 & 0.255 & 0.348 \\
\hline R5 & 0.276 & 0.244 & 0.276 & 0.414 & 0.222 & 0.126 \\
\hline R6 & 0.338 & 0.027 & 0.338 & 0.531 & 0.493 & 0.628 \\
\hline R7 & 0.972 & 1.301 & 0.972 & 0.143 & 0.184 & 0.352 \\
\hline $\mathrm{R} 8$ & 1.024 & 1.112 & 1.024 & 0.995 & 0.591 & 0.694 \\
\hline R9 & 0.605 & 0.603 & 0.605 & 0.215 & 0.217 & 0.117 \\
\hline $\mathrm{R} 10$ & 0.425 & 0.823 & 0.425 & 0.416 & 0.095 & 0.393 \\
\hline $\mathrm{R} 11$ & 1.056 & 0.982 & 1.056 & 0.155 & 0.325 & 0.388 \\
\hline $\mathrm{R} 12$ & 0.677 & 0.752 & 0.677 & 0.194 & 0.463 & 0.130 \\
\hline $\mathrm{R} 13$ & 0.809 & 0.976 & 0.810 & 0.210 & 0.324 & 0.453 \\
\hline $\mathrm{R} 14$ & 0.848 & 0.597 & 0.847 & 0.395 & 0.199 & 0.132 \\
\hline $\mathrm{R} 15$ & 1.146 & 0.845 & 1.146 & 0.464 & 0.353 & 0.081 \\
\hline
\end{tabular}

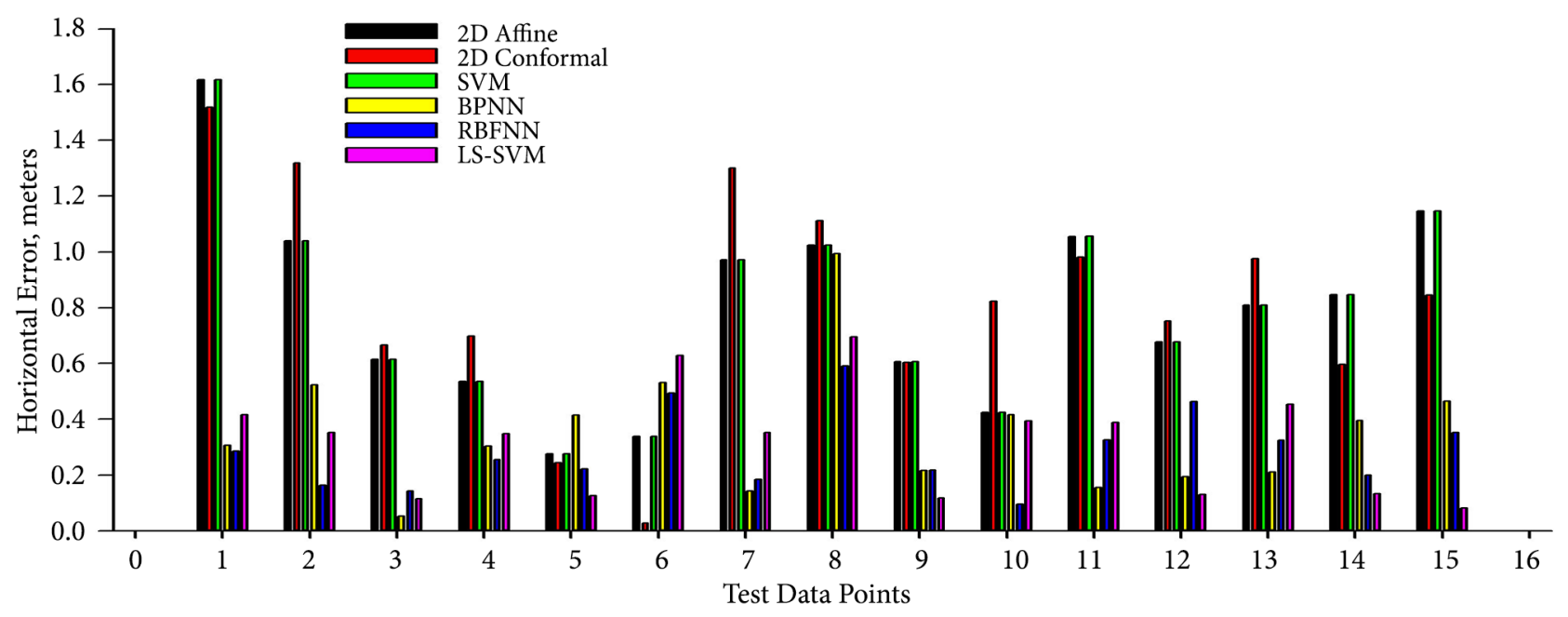

Figure 5. The horizontal positional error for the test data

attributed to the heavily reliance on several parameters ( $\varepsilon, C$, and kernel function parameter) which needed to be optimised by the modeller (Monien \& Decker, 2005). Hence, optimal combination of these parameters is sometimes a difficult task to carry out. These phenomena were observed during the SVM training where only the polynomial kernel of order 1 was able to perform better among the candidate kernel functions tested in this study. In relation to the conventional methods, the lack of computational adaptive strategy makes them rigid and thus could only produce better results based on the extent the formula could approximate the relationship between the input-output data to the underlying true function.

Summary statistics of the total horizontal coordinate residuals produced based on the test data is presented in Table 4.

In Table 4, the inference made with regards to the maximum error (Equation (20)) indicates the size of the residual distance that was achieved when the methods were applied within the study area. From Table 4, it is known that the LS-SVM, BPNN and RBFNN achieved the best maximum error among the candidate models. The AHE (Equation (21)) results in Table 4 quantify the average amount of horizontal positional dispersion. In Table 4 , it can be seen that the RBFNN furnished the least AHE value of $0.287 \mathrm{~m}$, followed by LS-SVM which had $0.315 \mathrm{~m}$. The BPNN, SVM, 2D affine and 2D conformal gave 0.354 , 0.779 and $0.831 \mathrm{~m}$ respectively. 
Table 4. Statistics of the total horizontal residuals across the testing data

\begin{tabular}{|l|c|c|c|c|c|c|}
\hline Performance Index & 2D affine & 2D conformal & SVM & BPNN & RBFNN & LS-SVM \\
\hline Max Error & 1.618 & 1.519 & 1.618 & 0.995 & 0.591 & 0.694 \\
\hline AHE & 0.799 & 0.831 & 0.799 & 0.354 & 0.287 & 0.315 \\
\hline SD & 0.357 & 0.397 & 0.357 & 0.23 & 0.14 & 0.194 \\
\hline RMS $_{\text {HE }}$ & 0.87 & 0.915 & 0.87 & 0.418 & 0.318 & 0.366 \\
\hline
\end{tabular}

In relation to the SD (Equation (22)) results in Table 4, the RBFNN achieved the best precision of $0.14 \mathrm{~m}$ followed by the LS-SVM which had $0.194 \mathrm{~m}$. The BPNN produced $0.230 \mathrm{~m}$ whereas the $2 \mathrm{D}$ affine and SVM gave identical precision of $0.357 \mathrm{~m}$. The $2 \mathrm{D}$ conformal model had a transformation precision of $0.397 \mathrm{~m}$. This implies that most of the LS-SVM and RBFNN transformation results are spread out and they are much closer to the mean value than the other methods. To further assess how well the transformed coordinates fit the observed data, the $\mathrm{RMS}_{\mathrm{HE}}$ (Eq. ((23)) was estimated. Quantitative evidence based on the $\mathrm{RMS}_{\mathrm{HE}}$ results (Table 4) have demonstrated that there is minimal local geodetic network distortion effect on the final transformed coordinates given by LS-SVM, RBFNN and BPNN.

The box and whisker plot (Figure 6) clearly demonstrates the statistical distributional characteristics of the horizontal positional errors produced by the various methods. It can be observed that the box plot for the LSSVM, RBFNN and BPNN is comparatively shorter than the SVM, 2D affine and conformal models. This suggests that the overall estimated horizontal residuals produced by the LS-SVM, RBFNN and BPNN has a high level of agreement with each other. The SVM, 2D affine and 2D conformal box plots (Figure 6) suggest the existence of higher variability in the horizontal residual distribution as compared with the other methods. This can be seen from Figure 6 where the interquartile range is smaller for the LS-SVM, RBFNN and BPNN. From a practical point of view, the testing results produced by the proposed LSSVM and the widely used BPNN and RBFNN are satisfactory for cadastral surveying applications in Ghana. This statement is buttressed by the $\pm 0.914 \mathrm{~m}$ tolerance residual distance set by the Ghana Survey and Mapping Division of Lands Commission for its cadastral surveying works (Yakubu \& Kumi-Boateng, 2015; Ziggah, Youjian, Laari, \& Hui, 2017). Therefore, the quantitative results presented in Table 4 affirm that assertion.

\section{Conclusions}

In this paper, the coordinate transformation strength of LS-SVM has been examined for the first time. The method was applied and tested in the Ghana geodetic reference network and the results compared with SVM and the widely used BPNN, RBFNN, 2D affine, and 2D conformal techniques. The transformation results of this study showed that the LS-SVM could perform creditably well to the RBFNN but outperformed all the other methods. Additionally, the LS-SVM results in this research suggest it usefulness for cadastral surveying applications in Ghana. That is, the LS-SVM achieved a tolerance residual distance below the $\pm 0.914 \mathrm{~m}$ set by the Ghana Survey and Mapping Division of Lands Commission. Therefore, it can be proposed that the LS-SVM model could be useful means for coordinate transformation in any of the area of interest considered in this study. To conclude, the results obtained show that the proposed LS-SVM has demonstrated

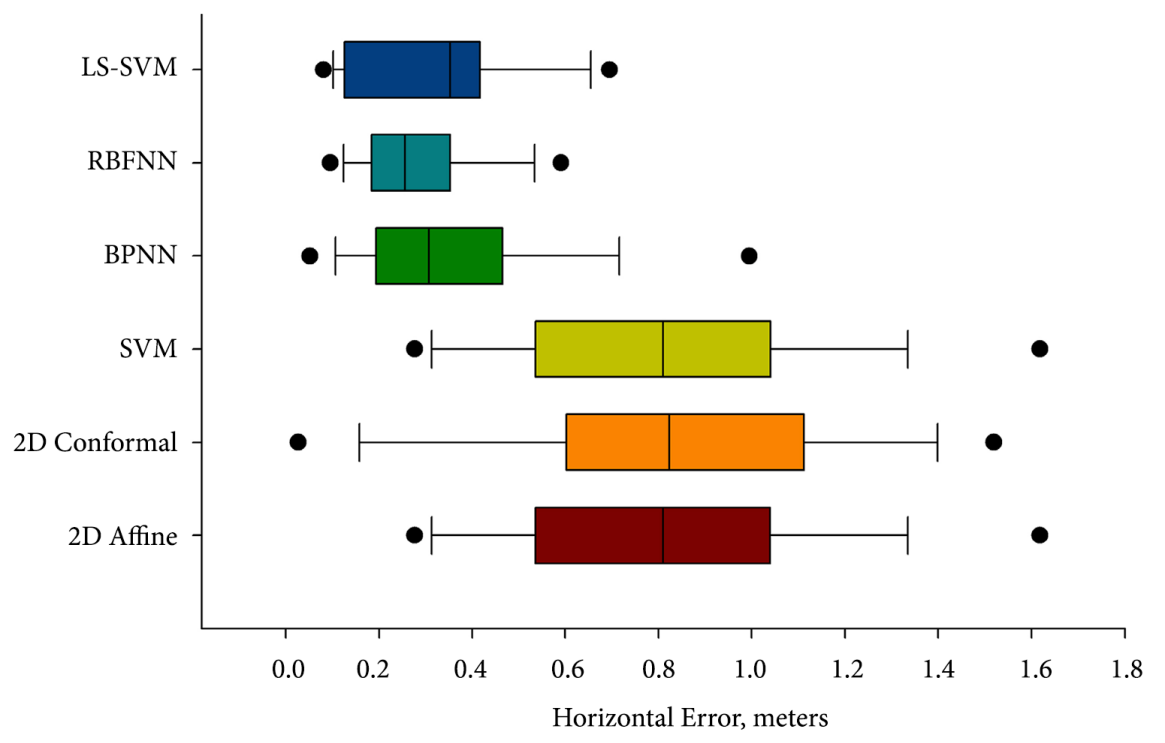

Figure 6. Horizontal positional error variation based on the testing data 
promising indication of calibration power and good generalisation performance in the coordinate transformation. Hence, the proposed LS-SVM model has provided enough convincing evidence to confidently be used for future research works in coordinate transformation.

\section{Acknowledgements}

The authors acknowledge the support from the Ghana Survey and Mapping Division of Lands Commission for making available to us the geodetic triangulation network data of Ghana.

\section{Author contributions}

YYZ conceived the research concept and design, data analysis and interpretation, and writing of the paper. $\mathrm{YH}$ supervised the entire work and final approval of the work. YI collected and compiled the data for the processing. PBL carried out critical revision of the paper from the draft stage to the final output.

\section{Disclosure statement}

The authors have no competing financial, professional, or personal interests from other parties.

\section{References}

Ao, S. I., \& Palade, V. (2011). Ensemble of Elman neural networks and support vector machines for reverse engineering of gene regulatory networks. Applied Soft Computing, 11, 17181726. https://doi.org/10.1016/j.asoc.2010.05.014

Ayer, J., \& Fosu, C. (2008). Map coordinates referencing and the use of GPS datasets in Ghana. Journal of Science and Technology, 28, 116-127.

Ayer, J. (2008). Transformation models and procedures for framework integration of Ghana geodetic network. The Ghana Surveyor, 1, 52-58.

Bishop, C. M. (1995). Neural networks for pattern recognition. UK: Oxford Press.

Chang, N. B., Han, M., Yao, W., Chen, L. C., \& Xu, S. (2010). Change detection of land use and land cover in an urban region with SPOT-5 images and partial Lanczos extreme learning machine. Journal of Applied Remote Sensing, 4(1), 11-15.

Deo, R. C., \& Şahin, M. (2016). An extreme learning machine model for the simulation of monthly mean streamflow water level in eastern Queensland. Environmental monitoring and assessment, 188(2), 1-24.

https://doi.org/10.1007/s10661-016-5094-9

Deo, R. C., Tiwari, M. K., Adamowski, J. F., \& Quilty, J. M. (2017). Forecasting effective drought index using a wavelet extreme learning machine (W-ELM) model. Stochastic Environmental Research and Risk Assessment, 31(5), 1211-1240. https://doi.org/10.1007/s00477-016-1265-Z

Drucker, H., Burges, C. J. C., Kaufman, I., Smola, A., \& Vapnik, V. (1997). Support vector regression machines. In M. Mozer, M. Jordan, \& T. Petsche (Eds.), Advances in Neural Information Processing Systems, 9, 155-161. Cambridge, MA: MIT Press.
Durmaz, M., \& Karslioglu, M. O. (2011). Non-parametric regional VTEC modeling with Multivariate Adaptive Regression B-Splines. Advances in Space Research, 48(9), 1523-1530. https://doi.org/10.1016/j.asr.2011.06.031

Farag, A., \& Mohamed, R. M. (2004). Regression using support vector machines: Basic foundation. Technical Report, University of Louisville.

Fosu, C., Poku-Gyamfi, Y., \& Hein, W. G. (2006). Global Navigation Satellite System (GNSS) - A utility for sustainable development in Africa. 5th FIG Regional Conference on Promoting Land Administration and Good Governance, Workshop AFREF I, Accra, Ghana (pp. 1-12).

Gencoglu, M. T., \& Uyar, M. (2009). Prediction of flashover voltage of insulators using least squares support vector machines. Expert Systems with Applications, 36, 10789-10798. https://doi.org/10.1016/j.eswa.2009.02.021

Ghilani, C. (2010). Adjustment computations: Spatial Data Analysis $\left(5^{\text {th }}\right.$ ed.). New York, USA: John Wiley and Sons Inc.

Gullu M., Yilmaz M., Yilmaz, I., \& Turgut, B. (2011). Datum transformation by artificial neural networks for geographic information systems applications. International Symposium on Environmental Protection and Planning: Geographic Information Systems (GIS) and Remote Sensing (RS) Applications (ISEPP). Izmir-Turkey (pp. 13-19).

Gullu, M. (2010). Coordinate transformation by radial basis function neural network. Scientific Research and Essays, 5, 3141-3146.

Güraksin, G. E., Hakli, H., \& Harun, U. (2014). Support vector machines classification based on particle swarm optimization for bone age determination. Applied Soft Computing, 24, 597602. https://doi.org/10.1016/j.asoc.2014.08.007

Haykin, S. (1990). Neural networks: a comprehensive foundation. New Jersey, USA: Prentice-Hall.

Hornik, K., Stinchcombe, M., \& White, H. (1989). Multilayer feed forward networks are universal approximators. Neural Networks, 2, 359-366. https://doi.org/10.1016/0893-6080(89)90020-8

Huang, F. M., Wu, P., \& Ziggah, Y. Y. (2016). GPS monitoring landslide deformation signal processing using time-series model. International Journal of Signal Processing, Image Processing and Pattern Recognition, 9(3), 321-332. https://doi.org/10.14257/ijsip.2016.9.3.28

Kavzoglu, T., \& Saka, M. H. (2005). Modelling local GPS/levelling geoid undulations using artificial neural networks. Journal of Geodesy, 78(9), 520-527. https://doi.org/10.1007/s00190-004-0420-3

Kecman, V. (2001). Learning and soft computing. A Bradford Book. Massachusetts, USA: The MIT Press.

Konakoğlu, B., Cakir, L., \& Gökalp, E. (2016). 2D coordinates transformation using artificial neural networks. Geo Advances 2016: ISPRS Workshop on Multi-dimensional \& Multi-scale Spatial Data Modeling, At Mimar Sinan Fine Arts University/ Istanbul, Volume XLII-2/W1: 3rd International GeoAdvances Workshop, 2016.

Konakoğlu, B., \& Gökalp, E. (2016). A Study on 2D similarity transformation using multilayer perceptron neural networks and a performance comparison with conventional and robust outlier detection methods. Acta Montanistica Slovaca, 21(4), 324-332.

Kotzev, V. (2013). Consultancy service for the selection of a new projection system for Ghana. Draft Final Reports, World Bank Second Land Administration Project (LAP-2), Ghana.

Kumi-Boateng, B., \& Ziggah, Y. Y. (2017). Horizontal coordinate 
transformation using artificial neural network technology - A case study of Ghana geodetic reference network. Journal of Geomatics, 11(1), 1-11.

Li, X. Z., \& Kong, J. M. (2014). Application of GA-SVM method with parameter optimization for landslide development prediction. Natural Hazards and Earth System Sciences, 14(3), 525-533. https://doi.org/10.5194/nhess-14-525-2014

Lin, L. S., \& Wang, Y. J. (2006). A study on cadastral coordinate transformation using artificial neural network. Proceedings of the $27^{\text {th }}$ Asian Conference on Remote Sensing (pp. 1-6). Ulaanbaatar, Mongolia.

Mihalache, R. M. (2012). Coordinate transformation for integrating map information in the new geocentric European system using artificial neural networks. GeoCAD, 1-8.

Mohammadi, K., Shamshirband, S., Motamedi, S., Petković, D., Hashim, R., \& Gocic, M. (2015). Extreme learning machine based prediction of daily dew point temperature. Computers and Electronics in Agriculture, 117, 214-225. https://doi.org/10.1016/j.compag.2015.08.008

Monien, K., \& Decker, R. (2005). Strengths and weaknesses of support vector machines within marketing data analysis. In Innovations in Classification, Data Science, and Information Systems (pp. 355-362). Berlin, Heidelberg: Springer. https://doi.org/10.1007/3-540-26981-9_41

Mugnier, J. C. (2000). OGP-Coordinate conversions and Transformations including formulae. COLUMN, Grids and Datums, The Republic of Ghana. Photogrammetric Engineering and Remote Sensing, 695-697.

Okwuashi, O., \& Ndehedehe, C. (2015). Digital terrain model height estimate support vector machine regression. South African Journal of Science, 111(9/10), 1-5. https://doi.org/10.17159/sajs.2015/20140153

Okwuashi, O., \& Ndehedehe, C. (2017). Tide modelling using support vector machine regression. Journal of Spatial Science, 62(1), 29-46.

Pal, M. (2009). Extreme-learning-machine-based land cover classification. International Journal of Remote Sensing, 30(14), 3835-3841. https://doi.org/10.1080/01431160902788636

Park, J., \& Sandberg, I. W. (1991). Universal approximation using radial basis function networks. Neural Computation, 3(2), 246-257. https://doi.org/10.1162/neco.1991.3.2.246

Poku-Gyamfi, Y. (2009). Establishment of GPS Reference Network in Ghana (PhD Dissertation). Universitat der Bundeswehr Munchen, Germany.

Smola, A., \& Schölkopf, B. (2004). A tutorial on support vector regression. Statistics and Computing, 14, 199-222. https://doi.org/10.1023/B:STCO.0000035301.49549.88

Sorkhabi, O. M. (2015). Geoid determination based on log sigmoid function of artificial neural networks (A case study: Iran). Journal of Artificial Intelligence in Electrical Engineering, 3(12), 18-24.

Suykens, J. A. K, Vandewalle, J. (1999). Least square support vector machine classifiers. Neural Processing Letters, 9, 293-300. https://doi.org/10.1023/A:1018628609742

Suykens, J. A. K., Van Gestel, T., De Brabanter, J., De Moor, B., \&
Vandewalle, J. (2002). Least squares support vector machines. World Scientific, Singapore. https://doi.org/10.1142/5089

Tierra, A., Dalazoana, R., \& De Freitas, S. (2008). Using an artificial neural network to improve the transformation of coordinates between classical geodetic reference frames. Computers \& Geosciences, 34, 181-189.

https://doi.org/10.1016/j.cageo.2007.03.011

Tierra, A., \& Romero, R. (2014). Planes coordinates transformation between PSAD56 to SIRGAS using a Multilayer Artificial Neural Network. Geodesy and Cartography, 63, 199-209. https://doi.org/10.2478/geocart-2014-0014

Tierra, A. R., De Freitas, S. R. C., \& Guevara, P. M. (2009). Using an artificial neural network to transformation of coordinates from PSAD56 to SIRGAS95. In H. Drewes (Ed.), Geodetic reference frames. International Association of Geodesy Symposia, 134, 173-178. Germany: Springer-Verlag Berlin Heidelberg. https://doi.org/10.1007/978-3-642-00860-3_27

Tiwari, M., Adamowski, J., \& Adamowski, K. (2016). Water demand forecasting using extreme learning machines. Journal of Water and Land Development, 28(1), 37-52. https://doi.org/10.1515/jwld-2016-0004

Turgut, B. (2010). A Back-propagation artificial neural network approach for three-dimensional coordinate transformation. Scientific Research and Essays, 5, 3330-3335.

Turgut, B. (2016). Application of back propagation artificial neural networks for gravity field modelling. Acta Montanistica Slovaca, 21(3), 200-207.

Vapnik, V. N. (1998). Statistical learning theory. New York, USA: John Wiley \& Sons.

Varga, M., Grgić, M., \& Bašić, T. (2015). Empirical comparison of the geodetic coordinate transformation models: a case study of Croatia. Survey Review, 1-13.

Xiang-Yang, W., Jing-Wei, C., \& Hong-Ying, Y. (2011). A New integrated SVM classifiers for relevance feedback contentbased image retrieval using EM parameter estimation. Applied Soft Computing, 11(2), 2787-2804.

https://doi.org/10.1016/j.asoc.2010.11.009

Yakubu, I., \& Kumi-Boateng, B. (2015). Ramification of datum and ellipsoidal parameters on postprocessed differential global positioning system (DGPS) data - A case study. Ghana Mining Journal, 15, 1-9.

Yang, Y. X. (2009). Chinese geodetic coordinate system 2000. Chinese Scientific Bulletin, 54, 2714-2721. https://doi.org/10.1007/s11434-009-0342-9

Yilmaz, I., \& Gullu, M. (2012). Georeferencing of historical maps using back propagation artificial neural network. Experimental Techniques, 36, 15-19.

https://doi.org/10.1111/j.1747-1567.2010.00694.x

Zaletnyik, P. (2004). Coordinate transformation with neural networks and with Polynomials in Hungary. International Symposium on Modern Technologies, Education and Professional Practice in Geodesy and Related Fields (pp. 471-479). Sofia, Bulgaria.

Ziggah, Y. Y., Youjian, H., Laari, P. B., \& Hui, Z. (2017). Novel approach to improve geocentric translation model performance using artificial neural network technology. Boletim de Ciências Geodesica, 23(1), 213-233.

https://doi.org/10.1590/s1982-21702017000100014

Ziggah, Y. Y., Youjian, H., Tierra, A., Konaté, A. A., \& Hui, Z. (2016). Performance evaluation of artificial neural networks for planimetric coordinate transformation - a case study, Ghana. Arabian Journal of Geosciences, 9(17), 1-16. https://doi.org/10.1007/s12517-016-2729-7 